\title{
Boundary Value Problems on Manifolds with Fibered Boundary
}

\author{
A. Savin, B. Sternin
}

\section{Introduction}

The modern theory of elliptic boundary value problems deals with two types of problems. First, one has classical boundary value problems that admit a realization as some bounded operators in Sobolev spaces. Secondly, there are boundary value problems of Atiyah-Patodi-Singer type that can be realized as Fredholm operators in some subspaces of the Sobolev spaces. Moreover, the subspaces in question are the ranges of some pseudodifferential projections acting in the Sobolev spaces. These two classes of boundary value problems have a substantial difference. Namely, the first class is defined only for some elliptic operators (defined on manifolds with boundary) and the corresponding obstruction in the stable theory is known as the Atiyah-Bott condition [1]. The second class of boundary value problems does not have this restriction: Fredholm boundary value problems of the above described type can be defined for an arbitrary elliptic operator. On the other hand, this type of boundary conditions imposes an essential restriction on the right-hand sides of the boundary value problem. Namely, it is supposed that the right-hand sides are taken from a subspace of the Sobolev space of a possibly infinite codimension. The following question naturally arises: is it possible to construct an elliptic theory, which is a deformation of the two theories such that on the one end it reduces to the theory of classical boundary value problems, while at the other end it gives the Atiyah-Patodi-Singer problems? In other words, the problem is to construct a series of intermediate theories of elliptic boundary value problems that would contain as particular (and in a sense polar) examples classical boundary value problems and the problems with projections. The present paper is devoted to this problem.

Clearly, the construction of such a theory requires some additional assumptions on the geometry of the boundary of the manifold, where the boundary value problems are considered. In the present paper we assume that the boundary is a fibration over a compact base with a compact fiber. In this setting, we establish all the analytical results we need (finiteness theorem, in particular). Moreover, we compute the obstruction to the existence of elliptic problems of this type. The obstruction turns out to be an element of $K^{1}$-group of the cotangent bundle of the base. This explains the nature of the topological 
Atiyah-Bott obstruction as well as the absence of obstruction for Atiyah-Patodi-Singer problems. Indeed, in the special case, when the base coincides with the boundary, one obtains classical boundary value problems. On the contrary, when the base of the fibration is a point, we obtain Atiyah-Patodi-Singer problems.

It should be mentioned that the importance and the interest of this class of boundary value problems on manifolds with fibered boundary is clear even in the case, when the boundary is a covering, i.e. a fibration with a discrete fiber. For a covering, the class of boundary value problems under consideration includes a number of nonlocal boundary value problems (see [2]). Rather surprisingly, the boundary conditions in the intermediate theories on manifolds with fibered boundary are defined by operators with discontinuous symbols.

Let us briefly describe the contents of the paper. We construct an algebra of operators with discontinuous symbols on a fibration in the first three sections. In particular, we establish the composition formula and prove the Fredholm criterion. Boundary value problems on manifolds with fibered boundary are defined in Section 5 . We prove the finiteness theorem and in Section 6 compute the topological obstruction to pose a Fredholm boundary value problem for an elliptic operator. Examples of boundary value problems for the Hirzebruch operator are presented.

We are grateful to Professor G. Rozenblioum of Chalmers University, Göteborg, Sweden for numerous helpful discussions and Dr. V. Nazaikinskii for help on a number of topics. The results were announced at the Conference "Workshop in Partial Differential Equations", Potsdam, Germany, November 12-16, 2001. We would like to thank the organizers of this conference for their hospitality and support. The work was partially supported by RFBF grants 00-01-00161, 01-01-06013, 99-01-01254. The paper was partially written at Chalmers University of Technology and supported by a grant from the Swedish Royal Academy of Sciences.

\section{Symbolic algebra}

Let $\pi: M \rightarrow X$ be a locally trivial fiber bundle of compact smooth manifolds $M$ and $X$ with a typical fiber $Y$. Manifolds $M, Y, X$ are assumed to be closed. The fiber $\pi^{-1}(x)$ over $x \in X$ is denoted by $Y_{x}$.

On the total space of the fibration, we consider special coordinates described as follows. For a domain $U \subset X$ with coordinates $x_{1}, \ldots, x_{n}, n=\operatorname{dim} X$ and a trivializing mapping $\alpha_{U}$

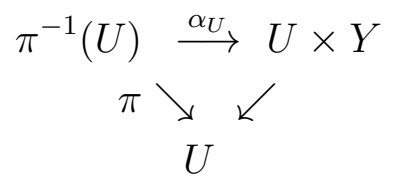


we consider some coordinates $y_{1}, \ldots, y_{m}, m=\operatorname{dim} Y$ in a domain $V \subset Y$ of the fiber. Denote the dual coordinates in the cotangent space by $\left(\xi_{1}, \ldots, \xi_{n}, \eta_{1}, \ldots, \eta_{m}\right)$.

Definition 1. Principal symbol of order zero is a function

$$
a_{M} \in C^{\infty}\left(T^{*} M \backslash \pi^{*} T^{*} X\right)
$$

which is homogeneous in covariables of order zero and is directionally smooth as covariables approach the plane $\pi^{*} T^{*} X \subset T^{*} M$. More precisely, in local coordinates we suppose that the function

$$
a(x, y, \xi, t \eta), \quad(x, y, \xi, t, \eta) \in U \times V \times\left(\mathbb{R}^{n} \backslash\{0\}\right) \times \mathbb{R}_{+} \times\left(\mathbb{R}^{m} \backslash\{0\}\right)
$$

extends smoothly up to $t=0$.

The following notation will be used for the directional limit

$$
\widetilde{a}_{M}(x, y, \xi, \eta)=\lim _{t \rightarrow 0} a_{M}(x, y, \xi, t \eta)
$$

of the principal symbol at the plane $\pi^{*} T^{*} X$. This function is defined if both $|\xi| \neq 0$ and $|\eta| \neq 0$. It is homogeneous of order zero with respect to $\xi$ and $\eta$.

It is clear that the restriction of the principal symbol to the cosphere bundle $S^{*} M$ can have discontinuities. However, our definition is equivalent to the requirement that the restriction of the principal symbol to $S^{*} M$ extends to a smooth function on a compact manifold $\overline{S^{*} M \backslash \pi^{*} S^{*} X}$ with boundary. This space is a compactification of $S^{*} M \backslash \pi^{*} S^{*} X$ obtained by attaching to it sequences $\left(x_{i}, y_{i}, \xi_{i}, \eta_{i}\right)$ converging to a point in $\pi^{*} S^{*} X$ such that the quotients $\eta /|\eta|$ converge as well. One can show that this manifold is diffeomorphic to $S^{*} M \backslash U_{\pi^{*} S^{*} X}$, where $U_{\pi^{*} S^{*} X}$ is an open tubular neighborhood of $\pi^{*} S^{*} X$.

Definition 2. Operator symbol of order zero is a function

$$
a_{X} \in C^{\infty}\left(T^{*} X \backslash\{0\}, \Psi^{0}(Y)\right)
$$

that at a point $(x, \xi) \in T^{*} X \backslash 0$ is a classical pseudodifferential operator of order zero in the fiber $Y_{x}$. The function $a_{X}$ is assumed to be homogeneous in covariable of order zero and smoothly depending on $(x, \xi)$. More precisely, $a_{X}(x, \xi)$ is a smooth function with values in a Fréchet space.

Remark 1. Let us recall the definition of the Fréchet structure on the space of classical pseudodifferential operators, see [3, 4]. The set of seminorms is defined as follows. Fix on $Y$ a quantization mapping $\sigma \mapsto \widehat{\sigma}$, defined for smooth homogeneous symbols $\sigma$. Then a classical $\psi D O A$ of order $d$ on $Y$ is equivalent to a sum

$$
A \sim \sum_{j \geq 0} \widehat{a}_{d-j}, \quad a_{d-j} \in C^{\infty}\left(S^{*} Y\right) .
$$


Here the symbols $a_{k}$ are homogeneous of degree $k$. While the equivalence means that

$$
A-\sum_{N \geq j \geq 0} \widehat{a}_{d-j} \in \Psi^{d-N-1}(Y) .
$$

In these terms, the following two systems of seminorms on the space $\Psi^{d}(Y)$ :

1) $\|A\|_{\alpha, j}=\left\|a_{d-j}\right\|_{\alpha}$, where $\alpha$ runs over a countable set of seminorms on $C^{\infty}\left(S^{*} Y\right)$;

2) $\|A\|_{s, N}=\left\|A-\sum_{0 \leq j \leq N-1} \widehat{a}_{d-j}\right\|_{H^{s}(Y) \rightarrow H^{s-(d-N)}(Y)}$. This is an operator norm;

define the Fréchet structure.

Definition 3. Symbols $a_{M}$ and $a_{X}$ are said to be compatible if

$$
\sigma\left(a_{X}\right)=\widetilde{a}_{M},
$$

i.e. the principal symbol of the operator symbol $a_{X}$ is equal to the limiting value $\widetilde{a}_{M}$ of the principal symbol at the horizontal bundle $\pi^{*} T^{*} X$.

Definition 4. By $\Sigma(M, \pi)$ denote the algebra of compatible pairs $\left(a_{M}, a_{X}\right)$ with a componentwise product. An element $\sigma \in \Sigma(M, \pi)$ is called a symbol on $M$.

Example 1. Let $B \in C^{\infty}\left(X, \Psi^{0}(Y)\right)$ be a smooth family of pseudodifferential operators $B_{x}$ of order zero in the fibers:

$$
B_{x}: C^{\infty}\left(Y_{x}\right) \rightarrow C^{\infty}\left(Y_{x}\right) .
$$

Then the pair

$$
(\sigma(B), B)
$$

is a symbol on $M$. Indeed, the principal symbol $\sigma(B)(x, y, \eta)$ is smooth for $|\eta| \neq 0$, while the rescaling as in (11) does not change the symbol:

$$
\sigma(B)(x, y, t \eta)=\sigma(B)(x, y, \eta),
$$

since $\sigma(B)$ is homogeneous of order zero. Thus, in this case $\widetilde{\sigma(B)}=\sigma(B)$ and the compatibility condition (2) is clearly satisfied.

Example 2. Let us now choose a smooth symbol $a \in C^{\infty}\left(T^{*} M \backslash\{0\}\right)$. Then the pair

$$
\left(a,\left.a\right|_{\pi^{*} T^{*} X}\right)
$$

where operator symbol $\left.a\right|_{\pi^{*} T^{*} X}=a(x, y, \xi, 0)$ acts as a multiplication operator, is a symbol in the sense of Definition $\mathbb{1}$

Denote by $\Sigma_{0} \subset \Sigma(M, \pi)$ the subalgebra multiplicatively generated by the symbols from Examples 1 and 2 . 
Proposition 1. $\Sigma_{0}$ is dense in $\Sigma(M, \pi)$, where $\Sigma(M, \pi)$ is equipped with the Fréchet structure as a subspace:

$$
\Sigma(M, \pi) \subset C^{\infty}\left(S^{*} M \backslash U_{\pi^{*} S^{*} X}\right) \oplus C^{\infty}\left(S^{*} X, \Psi^{0}(Y)\right) .
$$

Proof. 1) Let us show that the subalgebra of principal symbols corresponding to $\Sigma_{0}$ is dense.

Consider a principal symbol $\sigma(x, y, \xi, \eta)$. By a partition of unity argument, it suffices to approximate $\sigma$ over a neighborhood of an arbitrary point $\left(x_{0}, y_{0}\right) \in M$, where we can use local coordinates. In the domain $|\eta|>2 \varepsilon \sqrt{\xi^{2}+\eta^{2}}$, where $\sigma$ is smooth, it is equal to a smooth symbol

$$
\chi\left(\frac{|\eta|}{\sqrt{\xi^{2}+\eta^{2}}}\right) \sigma(x, y, \xi, \eta),
$$

where the chopping function $\chi(t)$ is zero for $t<\varepsilon$ and is 1 for $t>2 \varepsilon$. Thus, the main problem is to approximate the symbol for small $\eta$.

To this end, consider the function $\sigma\left(x, y, \xi^{\prime}, t \eta^{\prime}\right)$. It is smooth for

$$
\left|\xi^{\prime}\right|=\left|\eta^{\prime}\right|=1 \quad \text { and } t \in[0,100] \text {. }
$$

Hence, it can be approximated by a sum of products of functions depending on two subsets of variables $\left(x, \xi^{\prime}, t\right)$ and $\left(y, \xi^{\prime}\right)$ :

$$
\sigma\left(x, y, \xi^{\prime}, t \eta^{\prime}\right) \sim \sum_{j=1}^{N} a_{j}\left(x, \xi^{\prime}, t\right) b_{j}\left(y, \eta^{\prime}\right),
$$

where the terms are smooth functions for the parameter values as in (31). Thus, the original symbol is approximated for small $\eta$ by the expression

$$
\sigma(x, y, \xi, \eta) \sim \sum_{j=1}^{N} a_{j}\left(x, \frac{\xi}{|\xi|}, \frac{|\eta|}{|\xi|}\right) b_{j}\left(y, \frac{\eta}{|\eta|}\right)
$$

for $|\eta| \leq 100|\xi|$. Here the terms $b_{j}$ have the desired form as in Example 1 and they belong to the subalgebra $\Sigma_{0}$. However, the functions $a_{j}$ are not smooth at $\eta=0$ (they contain terms $|\eta|)$. Let us eliminate this singularity.

To this end, consider the Taylor expansion of $a_{j}\left(x, \xi^{\prime}, t\right)$ in $t$ at zero. We obtain

$$
a_{j}\left(x, \frac{\xi}{|\xi|}, \frac{|\eta|}{|\xi|}\right)=\sum_{k=0}^{N^{\prime}} \frac{\partial^{k}}{\partial t^{k}} a_{j}\left(x, \frac{\xi}{|\xi|}, 0\right)\left(\frac{|\eta|}{|\xi|}\right)^{k} \frac{1}{k !}+O\left(\frac{|\eta|^{N^{\prime}+1}}{|\xi|^{N^{\prime}+1}}\right) .
$$

\footnotetext{
${ }^{1}$ Here and in what follows we use the term approximation in the sense of Fréchet topologies on the corresponding spaces.
} 
For $k$ even the corresponding term $|\eta|^{k} /|\xi|^{k}$ is smooth for $|\xi| \neq 0$. However, for $k$ odd the quotient $|\eta| /|\xi|$ is not smooth. We represent it in the form

$$
\frac{|\eta|}{|\xi|}=\sum_{i} \frac{\varphi_{i}\left(\frac{\eta}{|\eta|}\right)|\eta|}{\eta_{i}} \frac{\eta_{i}}{|\xi|}
$$

where $\varphi_{i}$ denotes a partition of unity on the sphere $|\eta|=1$ such that $\eta_{i}$ does not vanish on the support of $\varphi_{i}$. In this sum, the factors on the left correspond to symbols from Example 1 , while the quotient $\eta_{i} /|\xi|$ is smooth and corresponds to a symbol from Example 2.

Now the approximation of the symbol $\sigma$ is defined as follows. First, we approximate $\sigma$ for $|\eta|<|\xi|$ by the formula (州. Then the Taylor expansion in (5) is written with the decomposition of nonsmooth factors as in (6). The error term in the Taylor expansion shows that for $|\eta|<4 \varepsilon|\xi|$ (for $\varepsilon$ sufficiently small), the approximation is good. Denote the corresponding approximating element by

$$
\sum_{\alpha} a_{\alpha}(x, \xi, \eta) b_{\alpha}(y, \eta)
$$

( $a_{\alpha}$ and $b_{\alpha}$ are homogeneous in $(\xi, \eta)$ and $\eta$, respectively). Then the expression

$$
\chi\left(\frac{|\eta|}{\sqrt{\xi^{2}+\eta^{2}}}\right) \sigma(x, y, \xi, \eta)+\sum_{\alpha}\left(\left[1-\chi\left(\frac{|\eta|}{\sqrt{\xi^{2}+\eta^{2}}}\right)\right] a_{\alpha}(x, \xi, \eta)\right) b_{\alpha}(y, \eta)
$$

approximates the initial principal symbol with a small error as desired. This approximation can now be globalized in $x$ and $y$ by a partition of unity.

2 ) Let us show that a compatible pair $\left(p_{M}, p_{X}\right)$ of a principal symbol and an operator symbol can be approximated by elements from the subalgebra $\Sigma_{0}$.

It follows from the previous part of the proof that we need to prove this for the trivial principal symbol $p_{M}=0$ only.

So, let us consider a symbol $\left(0, p_{X}\right)$ defined by an operator-valued function $p_{X}(x, \xi) \in$ $\Psi^{-1}\left(Y_{x}\right)$.

Similar to the above, let us approximate $p_{X}$ in a neighborhood of a point $\left(x_{0}, \xi_{0}\right) \in$ $S^{*} X$. There $p_{X}$ can be approximated by its Taylor expansion in $x$ and $\xi$. We write this for brevity as

$$
p_{X}(x, \xi)=\sum_{1 \leq j \leq N^{\prime}} p_{j, x_{0}, \xi_{0}}(x, \xi) B_{j}+O\left(\left|x-x_{0}\right|^{N}+\left|\xi-\xi_{0}\right|^{N}\right),
$$

where $B_{j}$ are pseudodifferential operators in the fibers and $p_{j, x_{0}, \xi_{0}}(x, \xi)$ are smooth (scalar) functions.

Taking a cover of $S^{*} M$ with sufficiently small charts $U_{\alpha}$ and using the corresponding partition of unity $\varphi_{\alpha}$, we obtain an approximation

$$
\sum_{\alpha, j} \varphi_{\alpha} p_{j, x_{\alpha}, \xi_{\alpha}}(x, \xi) B_{j}
$$


for $p_{X}$. If the charts $U_{\alpha}$ are chosen good enough (e.g., their diameters are less than $\varepsilon$ and every point belongs to at most $l+1$ charts, where $l$ is the dimension of $\left.S^{*} X\right)$ then this expression is close to $p_{X}$ in the Fréchet space $\Sigma(M, \pi)$. To end the proof, it suffices to show that a term of the form $\varphi p(x, \xi) B$ is equal to a composition of a smooth symbol and a symbol of a family of pseudodifferential operators. Indeed, take $\chi \in C^{\infty}(X)$ such that $\pi^{*} \chi \equiv 1$ in a neighborhood of the support of $\varphi$. Then

$$
\varphi p(x, \xi) B=[\varphi p(x, \xi)] \chi B .
$$

Here $\chi B$ is a family of $\psi D O$ in the fibers, while $\varphi p(x, \xi)$ is the value for $\eta=0$ of the smooth symbol

$$
\chi(|\eta|) \varphi(x, \xi) p(x, \xi)
$$

where $\chi(t)$ is zero for $|t|>2 \varepsilon$ and 1 for $|t|<\varepsilon$. This completes the proof of the proposition.

\section{Operators on fibered manifolds}

The aim of this section is to show that a symbol $\sigma \in \Sigma(M, \pi)$ defines an operator

$$
\widehat{\sigma}: C^{\infty}(M) \longrightarrow C^{\infty}(M)
$$

acting on $C^{\infty}$ functions on $M$. Let us construct it. For a decomposition $\sigma=\left(a_{M}, a_{X}\right)$ this operator is defined as

$$
\widehat{\sigma} u=\left(\widehat{a}_{M}+\widehat{a}_{X-\widehat{\widetilde{a}}_{M}}\right) u, \quad u \in C^{\infty}(M),
$$

where the first component $\widehat{a}_{M}$ denotes the usual quantization of the principal symbol, while the second corresponds to quantization of operator-valued symbols (note that there is a correction term $\widehat{\widetilde{a}}_{M}$ to the operator symbol. This will be described later on).

More precisely, $\widehat{a}_{M}$ is first defined over charts $U_{\alpha} \subset X$ by the formula

$$
A_{\alpha} u=\sum_{j} \frac{1}{(2 \pi)^{m+n}} \psi_{j}(y) \int_{T_{x, y}^{*} M} e^{i(x \xi+y \eta)} a_{M}(x, y, \xi, \eta) \widehat{\left(u \chi_{j}\right)}(\xi, \eta) d \xi d \eta
$$

where $\chi_{j}(y)$ is a partition of unity subordinate to an atlas in the fiber, $\psi_{j}$ is equal to 1 in a neighborhood of the support of $\chi_{j}$ and vanishes far from it. Finally, the hat denotes the Fourier transform in $x$ and $y$.

The operator symbol $\widehat{\widetilde{a}}_{M}$ is defined along the same lines

$$
\widehat{\widetilde{a}}_{M}(x, \xi) v=\frac{1}{(2 \pi)^{m}} \sum_{j} \psi_{j}(y) \int_{T_{y}^{*} Y_{x}} e^{i y \eta} \widetilde{a}_{M}(x, y, \xi, \eta) \widehat{\left(v \chi_{j}\right)}(\eta) d \eta, \quad v \in C^{\infty}\left(Y_{x}\right),
$$


quantizing only in the fiberwise variables. This definition uses the same data $\chi_{j}, \psi_{j}$ as (8).

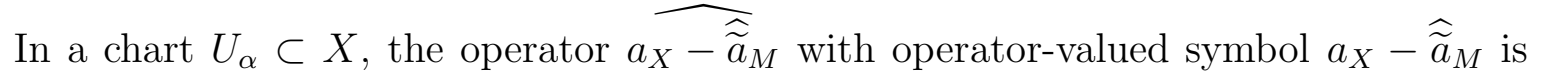
defined as

$$
B_{\alpha} u=\frac{1}{(2 \pi)^{n}} \int_{T_{x}^{*} X} e^{i x \xi}\left[a_{X}(x, \xi)-\widehat{\widetilde{a}}_{M}(x, \xi)\right] \widehat{u}(\xi) d \xi
$$

Here $\widehat{u}(\xi)$ denotes the Fourier transform in $x$ of $u(x, y)$.

Globally on $M$ the operator $\widehat{\sigma}$ is defined using the local expressions $A_{\alpha}, B_{\alpha}$ :

$$
\widehat{\sigma} u=\sum_{\alpha} \psi_{\alpha}^{\prime}\left(A_{\alpha}+B_{\alpha}\right)\left(\varphi_{\alpha}^{\prime} u\right)
$$

by a partition of unity $\chi_{\alpha}^{\prime}$ on $X$ subordinate to the atlas $\left\{U_{\alpha}\right\}$ and functions $\psi_{\alpha}^{\prime}$ supported in $U \alpha$ with the property $\psi_{\alpha}^{\prime} \chi_{\alpha}^{\prime}=\chi_{\alpha}^{\prime}$.

Remark 2. If $\sigma=\left(a,\left.a\right|_{\pi^{*} T^{*} X}\right)$ corresponds to a smooth symbol as in Example 2, then $\widehat{\sigma}$ is just the usual $\psi D O$ on $M$ with symbol $a$. On the other hand, for a family $B$ as in Example 1 consider the symbol $\sigma=(\sigma(B), B)$. Then one can prove that $\widehat{\sigma}=B$.

The quantization formula (77) resembles the formula for operators with discontinuous symbols on the circle, see [5].

Theorem 1. $\widehat{\sigma}$ extends to a bounded operator in Sobolev spaces $H^{s}(M), s \in \mathbb{R}$

$$
\widehat{\sigma}: H^{s}(M) \rightarrow H^{s}(M) .
$$

Proof. 1) The continuity in $H^{s}(M)$ of the first component (8) corresponding to the principal symbol was proved already in [6]. It was shown that no regularity of the symbol in covariables is required to obtain continuity in Sobolev spaces (for all $s$ ). Moreover, the norm of an operator with symbol $a\left(x^{\prime}, \xi^{\prime}\right)$ in $H^{s}(M)$ is bounded by the maximum of a finite number of derivatives in the geometric variables:

$$
\max _{|\alpha| \leq N} \sup _{\left(x^{\prime}, \xi^{\prime}\right) \in T^{*} M}\left|\partial_{x^{\prime}}^{\alpha} a\left(x^{\prime}, \xi^{\prime}\right)\right|
$$

up to a constant depending only on $s$ (the order $N$ of the derivatives also depends on $s$ ).

2) The continuity of operator (9) with operator-valued symbol in $L^{2}$ follows from the paper [7]. In this case the $L^{2}$-norm is also bounded by estimates of the form (11), where $a$ is replaced by the corresponding operator-valued symbol and the absolute value is replaces by the operator norm.

3) Let us prove that the term (9) defines a bounded operator in the Sobolev spaces. By interpolation it suffices to prove the boundedness for $s= \pm 2 m, m \in \mathbb{N}$. 
If $s=2 m$ then according to Theorem 7 of the Appendix an operator

$$
\widehat{q}_{X}: C^{\infty}(M) \rightarrow C^{\infty}(M)
$$

with operator-valued symbol $q_{X}(x, \xi) \in \Psi^{-1}\left(Y_{x}\right)$

$$
q_{X}=a_{X}-\widehat{\widetilde{a}}_{M}
$$

acts continuously in $H^{2 m}(M)$ if the composition

$$
\left(\triangle_{Y}+\xi^{2}\right)^{m} q_{X}(x, \xi)\left(\triangle_{Y}+\xi^{2}\right)^{-m}: C^{\infty}(Y) \rightarrow C^{\infty}(Y)
$$

is uniformly bounded in $L^{2}(Y)$, where $\triangle_{Y}$ is a positive Laplacian on $Y$.

The corresponding estimate

$$
\left\|\left(\xi^{2}+\triangle_{Y}\right)^{m} q_{X}(x, \xi)\left(\xi^{2}+\triangle_{Y}\right)^{-m}\right\|_{L^{2}(Y) \rightarrow L^{2}(Y)} \leq C
$$

can be proved using a decomposition of the commutator

$$
\left[\left(\triangle_{Y}+\xi^{2}\right)^{m}, q_{X}\right]=\sum_{-2 \leq j \leq 2 m-2} a_{j}(x, \xi)
$$

where the component $a_{j}$ has order $j$ and its norm in the spaces

$$
a_{j}(x, \xi): H^{s}(Y) \longrightarrow H^{s-j}(Y)
$$

is bounded by $(1+|\xi|)^{2 m-2-j}$. This decomposition can be proved by induction.

Using this decomposition, we can estimate each of the terms:

$$
\left\|a_{j}(x, \xi)\left(\xi^{2}+\triangle_{Y}\right)^{-m}\right\|_{L^{2}(Y) \rightarrow L^{2}(Y)} \leq\left\|a_{j}\right\|_{H^{j}(Y) \rightarrow H^{0}(Y)} \cdot\left\|\left(\triangle_{Y}+\xi^{2}\right)^{-m}\right\|_{H^{0}(Y) \rightarrow H^{j}(Y)} .
$$

Thus, we obtain

$$
\left\|a_{j}(x, \xi)\left(\xi^{2}+\triangle_{Y}\right)^{-m}\right\|_{L^{2}(Y) \rightarrow L^{2}(Y)} \leq(1+|\xi|)^{2 m-2-j} \cdot \max \left((1+|\xi|)^{-2 m+j},(1+|\xi|)^{-2 m}\right) .
$$

This expression is clearly uniformly bounded.

The remaining case $s=-2 m$ can be considered similarly.

\section{Operator algebra}

Theorem 2. The following composition formula is valid

$$
\widehat{\sigma}_{1} \widehat{\sigma}_{2}=\widehat{\sigma_{1} \sigma_{2}}+K
$$

where $\sigma_{1}, \sigma_{2} \in \Sigma(M, \pi)$ are symbols, while the error term $K$ is a compact operator in all spaces $H^{s}(M)$. Moreover, if $\sigma_{1} \in \Sigma_{0}$ then $K$ has order -1 in the scale. 
The proof of this theorem is done in the Appendix.

Definition 5. Denote by $\Psi^{0}(M, \pi)$ the space of operators of the form

$$
\widehat{\sigma}+K: C^{\infty}(M) \longrightarrow C^{\infty}(M),
$$

where operator $K$ extends to a compact operator in the scale $H^{s}(M)$.

The composition formula enables us to prove the following result.

Theorem 3. 1) $\Psi^{0}(M, \pi)$ is an algebra.

2) The subalgebra $\Psi_{0} \subset \Psi^{0}(M, \pi)$ generated by $\psi D O$ 's on $M$, families of $\psi D O$ 's in the fibers and compact operators is dense in $\Psi^{0}(M, \pi)$ with respect to operator norm in $H^{s}(M)$.

3) The norm, modulo compact operators, is equal to

$$
\inf _{k \in K\left(L^{2}(M)\right)}\|\widehat{\sigma}+k\|=\max \left(\sup _{(x, y, \xi, \eta) \in S^{*} M \backslash \pi^{*} S^{*} X}\left|a_{M}(x, y, \xi, \eta)\right|, \max _{(x, \xi) \in S^{*} X}\left\|a_{X}(x, \xi)\right\|\right),
$$

where $K\left(L^{2}(M)\right)$ denotes the ideal of compact operators.

Proof. 1) This straightforwardly follows from Theorem 2.

2) Suppose that $\widehat{\sigma} \in \Psi^{0}(M, \pi)$. According to Proposition 1 its symbol $\sigma$ can be approximated by a symbol $\sigma_{\varepsilon} \in \Sigma_{0}$. Then by Theorem 2 and Remark 2 we have $\widehat{\sigma}_{\varepsilon} \in \Psi_{0}$. On the other hand, the difference $\widehat{\sigma}-\widehat{\sigma}_{\varepsilon}=\widehat{\sigma-\sigma_{\varepsilon}}$ has a small symbol. Thus, its norm is small. This proves that $\Psi_{0}$ is dense in $\Psi^{0}(M, \pi)$.

3) By virtue of the second part of the theorem, it suffices to prove the estimate for $\widehat{\sigma} \in \Psi_{0}$ and $\sigma=\left(a_{M}, a_{X}\right)$.

Let us first prove the estimate from below. Suppose that $\left(x_{0}, y_{0}, \xi_{0}, \eta_{0}\right) \in T^{*} M \backslash \pi^{*} T^{*} X$. Then we choose a sequence of smooth functions

$$
u_{n}(x, y, t)=e^{i t\left(x \xi_{0}+y \eta_{0}\right)} \chi_{n}(x, y)
$$

where $\left\|\chi_{n}\right\|_{L^{2}(M)}=1$ and $\chi_{n}$ is supported in a ball of radius $1 / n$ around $\left(x_{0}, y_{0}\right)$.

Since $\widehat{\sigma} \in \Psi_{0}$, it has the form (modulo a compact operator)

$$
\widehat{\sigma}=\sum_{\alpha} \prod_{\beta} A_{\alpha \beta} B_{\alpha \beta}
$$

where $A_{\alpha \beta}$ are $\psi D O$ 's on $M$ and $B_{\alpha \beta}$ are families of $\psi D O$ 's in the fibers as in Example 1 .

Then as $t$ tends to infinity we obtain

$$
A_{\alpha \beta} u_{n}=\sigma\left(A_{\alpha \beta}\right)\left(x, y, \xi_{0}, \eta_{0}\right) u_{n}+O\left(\frac{1}{t}\right)
$$




$$
B_{\alpha \beta} u_{n}=\sigma\left(B_{\alpha \beta}\right)\left(x, y, \eta_{0}\right) u_{n}+O\left(\frac{1}{t}\right)
$$

according to the Hörmander's definition of pseudodifferential operators.

Hence, as $t \rightarrow \infty$ we obtain

$$
\widehat{\sigma} u_{n}=a_{M}\left(x, y, \xi_{0}, \eta_{0}\right) u_{n}+O\left(\frac{1}{t}\right) .
$$

On the other hand, $u_{n}$ weakly converges to zero as $t \rightarrow \infty$. Thus, for a compact operator $K$ we have $K u_{n} \rightarrow 0$. Therefore,

$$
(\widehat{\sigma}+K) u_{n}=a_{M}\left(x_{0}, y_{0}, \xi_{0}, \eta_{0}\right) u_{n}+u_{\varepsilon}+O\left(\frac{1}{t}\right)+K u_{n}
$$

where $u_{\varepsilon}=\left(a_{M}(x, y, \xi, \eta)-a_{M}\left(x_{0}, y_{0}, \xi_{0}, \eta_{0}\right)\right) u_{n}$, and for $n$ large we have $\left\|u_{\varepsilon}\right\|<\varepsilon$. For $t$ large this yields

$$
\left\|(\widehat{\sigma}+K) u_{n}\right\|_{L^{2}(M)} \geq\left|a_{M}\left(x_{0}, y_{0}, \xi_{0}, \eta_{0}\right)\right| \cdot\left\|u_{n}\right\|_{L^{2}(M)}-\varepsilon .
$$

Since $\varepsilon$ can be chosen arbitrarily small and $\left(x_{0}, y_{0}, \xi_{0}, \eta_{0}\right)$ and $K$ are also arbitrary, we have the desired estimate of the norm modulo compact operators

$$
\inf _{K}\|\widehat{\sigma}+K\| \geq \sup _{(x, y, \xi, \eta) \in T^{*} M \backslash \pi^{*} T^{*} X}\left|a_{M}(x, y, \xi, \eta)\right| .
$$

Let us prove the second part of the lower estimate that deals with the operator symbol. For $\left(x_{0}, \xi_{0}\right) \in S^{*} X$ and $v \in C^{\infty}\left(Y_{x_{0}}\right)$ consider the sequence

$$
u_{n}(x, y)=e^{i x \xi_{0} t} v(y) \chi_{n}(x)
$$

where $\chi_{n}(x),\left\|\chi_{n}\right\|_{L^{2}(X)}=1$ is a function on the base $X$ supported in a ball of radius $1 / n$ around $x_{0}$.

For $\widehat{\sigma}$ as in (15), we obtain

$$
A_{\alpha \beta} u_{n}=\sigma(A)\left(x, y, \xi_{0}, 0\right) u_{n}+O\left(\frac{1}{t}\right)
$$

and

$$
B_{\alpha \beta} u_{n}=B_{\alpha \beta} u_{n}
$$

By an argument similar to the previous part of the proof, we obtain

$$
\inf _{k \in K\left(L^{2}(M)\right)}\|\widehat{\sigma}+k\| \geq \max _{(x, \xi) \in S^{*} X}\left\|a_{X}(x, \xi)\right\|_{L^{2}\left(Y_{x}\right) \rightarrow L^{2}\left(Y_{x}\right)} .
$$


We now prove the estimate from above. This is done following the standard scheme (see, e.g. [8).

Let us denote by $\|\sigma\|$ the norm of the symbol $\sigma=\left(a_{M}, a_{X}\right)$, as defined by the right hand side of (14). Fix a constant $C>\|\sigma\|$. Then $C^{2}-\sigma^{*} \sigma$ is self-adjoint and positive symbol. Denote by $\sigma_{0}$ its positive square root

$$
C^{2}-\sigma^{*} \sigma=\sigma_{0}^{2} .
$$

Let us now approximate $\sigma_{0}$ by a symbol $\sigma_{\varepsilon} \in \Sigma_{0}$. Thus, according to the composition formula, we have for the corresponding operators

$$
C^{2}-\widehat{\sigma}^{*} \widehat{\sigma}=\widehat{\sigma}_{\varepsilon}^{*} \widehat{\sigma}_{\varepsilon}+K_{-1}+N_{\varepsilon}
$$

where $K_{-1}$ is a compact operator of order -1 in the Sobolev scale and $N_{\varepsilon}$ has norm less than $\varepsilon$. This formula gives the following estimate

$$
\|\widehat{\sigma} u\|_{L^{2}(M)}^{2} \leq C^{2}\|u\|_{L^{2}(M)}^{2}+\left\|K_{-1} u\right\|_{L^{2}(M)} \cdot\|u\|_{L^{2}(M)}+\varepsilon\|u\|_{L^{2}(M)}^{2} .
$$

Denote by $R_{\varepsilon}$ the smoothing operator on $L^{2}(M)$ with symbol

$$
\sigma\left(R_{\varepsilon}\right)(x, y, \xi, \eta)= \begin{cases}0, & \text { for }|\xi|^{2}+|\eta|^{2}>2 / \varepsilon \\ 1, & \text { for }|\xi|^{2}+|\eta|^{2}<1 / \varepsilon\end{cases}
$$

It is a compact operator and the following estimates are valid (see $[\mathbb{B}]$ )

$$
\left\|u-R_{\varepsilon} u\right\|_{L^{2}(M)} \leq\|u\|_{L^{2}(M)} \text { and } \quad\left\|u-R_{\varepsilon} u\right\|_{H^{-1}(M)} \leq c(\varepsilon)\|u\|_{L^{2}(M)},
$$

where $c(\varepsilon) \rightarrow 0$ as $\varepsilon \rightarrow 0$. Then (16) and (17) give the desired estimate:

$$
\left\|\widehat{\sigma}\left(u-R_{\varepsilon} u\right)\right\|_{L^{2}(M)}^{2} \leq C^{2}\|u\|_{L^{2}(M)}^{2}+C(\varepsilon)\|u\|_{L^{2}(M)}^{2}
$$

with $C(\varepsilon) \rightarrow 0$ as $\varepsilon \rightarrow 0$. Hence, if we take the compact operator $k=\widehat{\sigma} R_{\varepsilon}$, this yields for the infinum of the norm the following estimate

$$
\inf _{k \in K\left(L^{2}(M)\right)}\|\widehat{\sigma}+k\| \leq\|\sigma\|
$$

as desired.

Theorem is proved.

Denote by $\bar{\Psi}^{0}(M, \pi)$ the closure of $\Psi^{0}(M, \pi)$ with regard for the operator norm in $L^{2}(M)$. The next result describes the corresponding Calkin algebra. 


\section{Corollary 1.}

$$
\bar{\Psi}^{0}(M, \pi) / \mathcal{K} \simeq \bar{\Sigma}(M, \pi),
$$

where $\mathcal{K}$ denotes the ideal of compact operators and $\bar{\Sigma}(M, \pi)$ is the completion of the symbolic algebra $\Sigma(M, \pi)$ with respect to the norm $\|\cdot\|$. In more detail, the completion is a subalgebra

$$
\bar{\Sigma}(M, \pi) \subset C\left(S^{*} M \backslash U_{\pi^{*} S^{*} X}\right) \oplus C\left(S^{*} X, \bar{\Psi}^{0}(Y)\right),
$$

consisting of compatible pairs $\left(a_{M}, a_{X}\right)$ :

$$
\bar{\Sigma}(M, \pi)=\left\{a_{M} \oplus a_{X}\left|\quad a_{M}\right|_{\partial U_{\pi^{*} S^{*} X}}=\sigma\left(a_{X}\right)\right\},
$$

where $U_{\pi^{*} S^{*} X}$ denotes an open tubular neighborhood of $\pi^{*} S^{*} X$ in $S^{*} M$ and $\bar{\Psi}^{0}(Y)$ is the norm closure of the algebra of pseudodifferential operators of order zero in the fibers.

The proof of the corollary follows easily from the estimate modulo compact operators.

The description of the Calkin algebra enables us to state the Fredholm criterion.

Corollary 2. $\widehat{\sigma} \in \Psi^{0}(M, \pi)$ is a Fredholm operator in Sobolev spaces if and only if $\sigma$ is invertible.

Proof. The "if" part follows from the composition formula: the parametrix is given by $\widehat{\sigma^{-1}}$. The "only if" part is proved as follows. Let us assume at first that $s=0$. Suppose that $\widehat{\sigma}$ is a Fredholm operator in $L^{2}(M)$ with a left quasiinverse $A$ :

$$
A \widehat{\sigma}=1+K_{1} .
$$

Then this gives an apriori estimate

$$
\|u\| \leq C\|\widehat{\sigma} u\|+\left\|K_{1} u\right\| .
$$

If we substitute in this inequality the sequence $u_{n}(t)$, as in the proof of Theorem 5 , we obtain, choosing $n$ big enough and letting $t \rightarrow \infty$, that

$$
1 \leq C_{1}\left|\sigma_{M}\left(x_{0}, y_{0}, \xi_{0}, \eta_{0}\right)\right|
$$

and a similar estimate for the operator symbol

$$
1 \leq C_{2}\left\|\sigma_{X}\left(x_{0}, \xi_{0}\right)\right\|
$$

(these estimates can be obtained choosing approximations of $\widehat{\sigma}$ by elements of the subalgebra $\Psi_{0}$ ). Thus, the symbol is monomorphic. Passing to the adjoint operator, one proves the surjectivity.

Therefore, the symbol of a Fredholm operator is an isomorphism. The ellipticity of Fredholm operators in $H^{s}(M), s \neq 0$ can be proved along the same lines using the compositions $\triangle_{M}^{s / 2} \widehat{\sigma} \triangle_{M}^{-s / 2}$. 
Remark 3. A generalization of the composition formula and the finiteness theorem to operators acting in the sections of some vector bundles over $M$ is rather standard and is left to the reader, e.g. see [3].

\section{Elliptic Theory in subspaces}

Let us consider operators

$$
D: \operatorname{Im} P_{1} \longrightarrow \operatorname{Im} P_{2}
$$

acting in subspaces defined by projections

$$
P_{1}: C^{\infty}(M, E) \longrightarrow C^{\infty}(M, E), \quad P_{2}: C^{\infty}(M, F) \longrightarrow C^{\infty}(M, F),
$$

where $E, F$ are vector bundles over $M$. We suppose that $P_{1,2}$ belong to the algebra defined in previous sections: $P_{1,2} \in \Psi^{0}(M, \pi)$ and operator $D$ is a restriction of some operator $\widetilde{D}$

$$
\widetilde{D}: C^{\infty}(M, E) \longrightarrow C^{\infty}(M, F),
$$

also from our algebra: $\widetilde{D} \in \Psi^{0}(M, \pi)$.

Theorem 4. Operator (18) defines a Fredholm operator in Sobolev spaces if and only if the following two conditions are satisfied:

1) the principal symbol

$$
\sigma_{M}(D): \operatorname{Im} \sigma_{M}\left(P_{1}\right) \rightarrow \operatorname{Im} \sigma_{M}\left(P_{2}\right)
$$

is invertible over $S^{*} M \backslash \pi^{*} S^{*} X$;

2) the operator symbol

$$
\sigma_{X}(D): \operatorname{Im} \sigma_{X}\left(P_{1}\right) \rightarrow \operatorname{Im} \sigma_{X}\left(P_{2}\right)
$$

is invertible over $S^{*} X$.

Remark 4. In more detail, these conditions require that the principal symbol is a vector bundle isomorphism and the operator symbol is an invertible family of operators in subspaces defined by pseudodifferential projections (see, e.g., 90). Let us also mention that the symbol of a projection is a projection itself.

Proof. If the two symbols are invertible then the parametrix has the form:

$$
D^{-1}=P_{1} \widehat{\sigma^{-1}}: \operatorname{Im} P_{2} \longrightarrow \operatorname{Im} P_{1}
$$


where $\sigma=\left(\sigma_{M}(D), \sigma_{X}(D)\right)$ is the symbol of $D$. The fact that the differences $D^{-1} D-1$ and $D D^{-1}-1$ are compact operators follows from the composition formula.

Let us prove the "only if" part. If $D$ is a Fredholm operator, then the direct sum

$$
\operatorname{Im} P_{1} \oplus \operatorname{Im} P_{1}^{\perp} \stackrel{D^{*} D \oplus 1}{\longrightarrow} \operatorname{Im} P_{1} \oplus \operatorname{Im} P_{1}^{\perp}
$$

also has the Fredholm property. Moreover, there is an obvious isomorphism

$$
\operatorname{Im} P_{1} \oplus \operatorname{Im} P_{1}^{\perp} \simeq H^{s}(M, E) .
$$

Hence, $D^{*} D \oplus 1$ is an elliptic operator and its symbol has trivial kernel. The surjectivity of the symbol is proved along the same lines using the composition $D D^{*}$.

Remark 5. Consider a subclass of elliptic operators in subspaces (18), where both projections $P_{1}, P_{2}$ correspond to families of pseudodifferential projections in the fibers. Then for the identity map $\pi=$ id $: M \rightarrow M$ we obtain the usual operators on vector bundle sections over $M$, while for the collapsing map $\pi: M \rightarrow p t$ this construction gives the class of operators acting in subspaces defined by pseudodifferential projections (see [9]).

\section{Boundary value problems on manifolds with fibered boundary}

1. Main definitions. Consider $M$ a compact smooth manifold with boundary denoted by $\partial M$. Assume that $\partial M$ is the total space of a locally trivial fiber bundle

$$
\pi: \partial M \longrightarrow X
$$

with a compact base $X$ and a compact fiber $Y$ as in the previous sections.

On $M$ we consider an elliptic differential operator

$$
D: C^{\infty}(M, E) \longrightarrow C^{\infty}(M, F)
$$

of order $d$. To define the boundary conditions for $D$, we introduce the operator

$$
j: C^{\infty}(M, E) \longrightarrow C^{\infty}\left(M,\left.E\right|_{\partial M} ^{d}\right),
$$

that maps a function to its jet of order $d$ in the normal direction to the boundary:

$$
j u=\left(\left.u\right|_{\partial M},-\left.i \frac{\partial}{\partial t} u\right|_{\partial M}, \ldots,\left.\left(-i \frac{\partial}{\partial t}\right)^{d-1} u\right|_{\partial M}\right) .
$$


Here $t \geq 0$ denotes the normal coordinate near the boundary. We consider boundary value problems of the following form:

$$
\left\{\begin{array}{lll}
D u=f, & u \in C^{\infty}(M, E), & f \in C^{\infty}(M, F), \\
B j u=g \in \operatorname{Im} P, & \operatorname{Im} P \subset C^{\infty}(\partial M, G), & G \in \operatorname{Vect}(\partial M),
\end{array}\right.
$$

where the subspace $\operatorname{Im} P$ in the space of sections of a vector bundle $G$ is defined by a family of pseudodifferential projections over $X$ acting on functions in the fibers of $\pi$.

If $D$ is a first-order operator, then the boundary condition $B$ is assumed to be an element of the algebra $\Psi^{0}(\partial M, \pi)$. For operators of higher order the boundary condition is more complicated. In this case it has $d$ components

$$
B: C^{\infty}\left(\partial M,\left.E\right|_{\partial M} ^{d}\right) \rightarrow C^{\infty}(\partial M, G)
$$

and is defined as a composition

$$
B=\left(B_{0}, B_{1} \triangle_{\partial M}^{-1 / 2}, \ldots, B_{d-1} \triangle_{\partial M}^{-\frac{d-1}{2}}\right),
$$

where $\triangle_{\partial M}$ is a positive Laplacian on $\partial M$ and the components $B_{j}$ belong to $\Psi_{0}(\partial M, \pi)$.

Remark 6. This construction reduces to some well-known classes of boundary value problems for special types of projections $\pi$ :

1) $\pi=\mathrm{id}: \partial M \rightarrow \partial M$. In this case we obtain classical boundary value problems;

2) $\pi: \partial M \rightarrow p t$. This gives boundary value problems in subspaces (see [10]);

3) $\pi: \partial M \rightarrow X$ and $\pi$ is a covering. This gives a class of nonlocal boundary value problems studied in [2].

2. Finiteness theorem. The ellipticity condition of a boundary value problem for $D$ is formulated in terms of a special vector bundle

$$
L_{+}(D) \in \operatorname{Vect}\left(S^{*} \partial M\right)
$$

defined as a subbundle in the pull-back of $\left.E\right|_{\partial M} ^{d}$ to $S^{*} \partial M$ and generated by the Cauchy data at $t=0$ of functions $u(t)$ satisfying the ordinary differential equation

$$
\sigma(D)\left(x^{\prime}, 0, \xi^{\prime},-i \frac{d}{d t}\right) u(t)=0, \quad\left(x^{\prime}, \xi^{\prime}\right) \in S^{*} \partial M,
$$

that remain bounded as $t \rightarrow+\infty$ (see [11]). By $\widehat{L}_{+}(D)$ let us denote some subspace in $C^{\infty}\left(\partial M,\left.E\right|_{\partial M} ^{d}\right)$ that is defined by a pseudodifferential projection $Q$ on $\partial M$ with symbol projecting on the subbundle $\left.L_{+}(D) \subset E\right|_{\partial M} ^{d}$ along the complementary subbundle $L_{-}(D)$ corresponding to solutions decreasing at $-\infty$. Projections $Q$ are called Calderon projections for $D$.

The following Fredholm criterion is valid for boundary value problems (19). 
Theorem 5. Boundary value problem (19) defines a Fredholm operator for $s>d / 2$

$$
\left(\begin{array}{c}
D \\
B j
\end{array}\right): H^{s}(M, E) \longrightarrow \begin{gathered}
H^{s-d}(M, F) \\
P H^{s-1 / 2}(\partial M)
\end{gathered}
$$

if and only if two conditions are satisfied:

1) the principal symbol of $B$ is invertible on $S^{*} \partial M \backslash \pi^{*} S^{*} X$

$$
\sigma_{\partial M}(B): \operatorname{Im} \sigma_{\partial M}(Q) \longrightarrow \operatorname{Im} \sigma(P) ;
$$

2) the operator symbol of $B$ is invertible on $S^{*} X$ :

$$
\sigma_{X}(B): \operatorname{Im} \sigma_{X}(Q) \longrightarrow \operatorname{Im} P .
$$

Here $Q$ is a Calderon projection for $D$ and for $d>1$ we denote by $\sigma_{\partial M}(B)$ and $\sigma_{X}(B)$ the symbols of the tuple $\left(B_{0}, \ldots, B_{d-1}\right)$.

Remark 7. It should be noted that the conditions of the theorem use only the principal symbol $\sigma(D)$, symbol of $B$ and the projection $P$.

Proof. By the results of [10], the boundary value problem has the Fredholm property if and only if an operator on the boundary

$$
\left.\left(B_{0}, B_{1}, \ldots, B_{d-1}\right)\right|_{\operatorname{Im} Q}: \operatorname{Im} Q \rightarrow \operatorname{Im} P
$$

has the Fredholm property. We apply the Fredholm criterion stated in Theorem $₫$ to this operator in subspaces.

This readily shows that conditions 1 ) and 2) of the present theorem are necessary and sufficient for the Fredholm property to be valid.

Example 3. (Elliptic boundary value problem for the Hirzebruch operator). Let $M^{4 k}$ be an oriented $4 k$-dimensional manifold with boundary. Suppose that the boundary is a product

$$
\partial M=X^{e v} \times Y^{o d d}
$$

In a neighborhood of the boundary choose a metric corresponding to the Cartesian product $[0,1) \times X \times Y$. Consider the Hirzebruch operator

$$
D_{M}=d+\delta: \Lambda^{+}(M) \longrightarrow \Lambda^{-}(M)
$$


on $M$, where $d$ and $\delta$ are the exterior derivative and its adjoint and $\Lambda^{ \pm}(M)$ are the spaces of dual and antiselfdual forms on $M$ (e.g., see [12]). Then near the boundary $D_{M}$ can be represented in the form

$$
\frac{\partial}{\partial t}+\left(\begin{array}{cc}
D_{Y} & D_{X}^{*} \\
D_{X} & -D_{Y}
\end{array}\right)
$$

where $D_{X}$ denotes the Hirzebruch operator on $X$

$$
D_{X}: \Lambda^{+}(X) \longrightarrow \Lambda^{-}(X)
$$

and $D_{Y}$ denotes the odd analog of the Hirzebruch operator on Y (see [13]):

$$
\begin{gathered}
D_{Y}: \Lambda^{*}(Y) \longrightarrow \Lambda^{*}(Y), \\
D_{Y}=\tau\left(d_{Y}+\delta_{Y}\right),\left.\quad \tau\right|_{\Lambda^{p}(Y)}=i^{(\operatorname{dim} Y+1) / 2+p(p-1)} *, \quad \tau^{2}=i d .
\end{gathered}
$$

It is elliptic and self-adjoint. Denote by $\Pi_{+}$

$$
\Pi_{+}: \Lambda^{*}(Y) \longrightarrow \Lambda^{*}(Y)
$$

the nonnegative spectral projection of $D_{Y}$ and $\Pi_{-}$denote the complementary projection.

Consider the boundary value problem

$$
\left\{\begin{array}{l}
{\left[\frac{\partial}{\partial t}+\left(\begin{array}{cc}
D_{Y} & D_{X}^{*} \\
D_{X} & -D_{Y}
\end{array}\right)\right]\left(\begin{array}{l}
u \\
v
\end{array}\right)=\left(\begin{array}{l}
f_{1} \\
f_{2}
\end{array}\right),} \\
\left.\Pi_{+} u\right|_{\partial M}=g_{1} \in \operatorname{Im} \Pi_{+} \\
\left.\Pi_{-} v\right|_{\partial M}=g_{2} \in \operatorname{Im} \Pi_{-} .
\end{array}\right.
$$

Proposition 2. Boundary value problem (21) has the Fredholm property.

Proof. 1) According to Theorem 5, it suffices to check the invertibility of the corresponding principal and operator symbols.

2) An elementary computation shows that the principal symbol of the Calderon projection $Q$ on $S^{*} \partial M$ is the matrix

$$
\sigma_{\partial M}(Q)=\frac{1}{2}\left(\begin{array}{cc}
1+\sigma\left(D_{Y}\right) & \sigma^{*}\left(D_{X}\right) \\
\sigma\left(D_{X}\right) & 1-\sigma\left(D_{Y}\right)
\end{array}\right) .
$$

Hence, the operator symbol of the Calderon projection is

$$
\sigma_{X}(Q)=\frac{1}{2}\left(\begin{array}{cc}
1 & \sigma^{*}\left(D_{X}\right) \\
\sigma\left(D_{X}\right) & 1
\end{array}\right)
$$


We need to prove that the two maps

$$
\begin{aligned}
& \operatorname{Im} \sigma_{\partial M}(Q) \stackrel{\pi_{+} \oplus \pi_{-}}{\longrightarrow} \operatorname{Im}\left(\pi_{+} \oplus \pi_{-}\right), \\
& \operatorname{Im} \sigma_{X}(Q) \stackrel{\Pi_{+} \oplus \Pi_{-}}{\longrightarrow} \operatorname{Im} \Pi_{+} \oplus \operatorname{Im} \Pi_{-}
\end{aligned}
$$

are isomorphisms (here $\pi_{ \pm}$denote the principal symbols of $\Pi_{ \pm}$). To prove that the two maps are isomorphisms one can compute their compositions with the maps in the opposite direction

$$
\begin{aligned}
& \operatorname{Im}\left(\pi_{+} \oplus \pi_{-}\right) \stackrel{\sigma_{\partial M}(Q)}{\longrightarrow} \operatorname{Im} \sigma_{\partial M}(Q), \\
& \operatorname{Im} \Pi_{+} \oplus \operatorname{Im} \Pi_{-} \stackrel{\sigma_{X}(Q)}{\longrightarrow} \operatorname{Im} \sigma_{X}(Q) .
\end{aligned}
$$

An explicit computation shows that the compositions

$$
\left(\pi_{+} \oplus \pi_{-}\right) \sigma_{\partial M}(Q), \quad \sigma_{\partial M}(Q)\left(\pi_{+} \oplus \pi_{-}\right), \quad\left(\Pi_{+} \oplus \Pi_{-}\right) \sigma_{X}(Q), \quad \sigma_{X}(Q)\left(\pi_{+} \oplus \pi_{-}\right)
$$

have trivial kernels. Thus, the boundary value problem (21) satisfies the assumptions of Theorem 5 and, consequently, has the Fredholm property.

\section{Topological obstruction}

There is an obstruction to define a Fredholm boundary value problem for a given elliptic operator $D$ on $M$.

Theorem 6. Suppose that an elliptic differential operator D on $M$ has a Fredholm boundary value problem of the form (19). Then the principal symbol $\sigma(D)$ at the boundary has the following property

$$
\pi_{!}\left[\left.\sigma(D)\right|_{\partial M}\right]=0
$$

where

$$
\left[\left.\sigma(D)\right|_{\partial M}\right] \in K\left(T^{*} \partial M \times \mathbb{R}\right) \simeq K^{1}\left(T^{*} \partial M\right)
$$

is the difference construction and

$$
\pi_{!}: K^{1}\left(T^{*} \partial M\right) \rightarrow K^{1}\left(T^{*} X\right)
$$

is the direct image mapping in $K$-theory under the projection $\pi$. 
Proof. 1) Let $(D, B, P)$ be an elliptic boundary value problem for $D$. This means that there is a Fredholm operator

$$
\left(B_{0}, B_{1}, \ldots, B_{d-1}\right): \operatorname{Im} Q \rightarrow \operatorname{ImP}
$$

where $Q$ denotes a Calderon projection as previously.

The existence of a Fredholm isomorphism (23) implies that the symbols of the two subspaces $\operatorname{Im} Q$ and $\operatorname{Im} P$ are homotopic.

More precisely, consider both $Q$ and $P$ as projections in the direct sum

$$
C^{\infty}\left(\partial M,\left.E\right|_{\partial M} ^{d}\right) \oplus C^{\infty}(\partial M, G)
$$

Then the homotopy of the principal symbols is defined as

$$
q_{\partial M, \varphi}=\sigma(Q) \cos ^{2} \varphi+\sigma(P) \sin ^{2} \varphi+2 \sigma(P) \sigma_{\partial M}(B) \sigma(Q) \sin \varphi \cos \varphi
$$

and a similar formula is valid for the operator symbol

$$
q_{X, \varphi}=\sigma_{X}(Q) \cos ^{2} \varphi+P \sin ^{2} \varphi+2 P \sigma_{X}(B) \sigma_{X}(Q) \sin \varphi \cos \varphi
$$

This is a homotopy of compatible symbols and we obviously have at $\varphi=0$

$$
q_{\partial M, 0}=\sigma(Q), \quad q_{X, 0}=\sigma_{X}(Q)
$$

and for $\varphi=\pi / 2$

$$
q_{\partial M, \pi / 2}=\sigma(P), \quad q_{X, \pi / 2}=P .
$$

2) On the other hand, let us represent the topological invariant in (22) in analytic terms. It is well known that the element $\left[\left.\sigma(D)\right|_{\partial M}\right] \in K^{1}\left(T^{*} \partial M\right)$ can be expressed in terms of the symbol of the Calderon projection for $D$ :

$$
\left[\left.\sigma(D)\right|_{\partial M}\right]=\left[(2 \sigma(Q)(x, y, \xi, \eta)-1) \sqrt{\xi^{2}+\eta^{2}}+i \tau\right] \in K\left(T^{*} \partial M \times \mathbb{R}\right),
$$

where the coordinates correspond to the fibration $\pi: \partial M \rightarrow X$ and $\tau \in \mathbb{R}$ denotes an additional variable. The element is understood in the sense of the difference construction, since it is an isomorphism outside a compact set.

Let us represent the element in Eq. (26) as the difference construction in the sense of [3] for a family of elliptic operators in the fibers, where the parameter space is the product $T^{*} X \times \mathbb{R}$. More precisely, we will define a family of elliptic operators parametrized by $B\left(T^{*} X \times \mathbb{R}\right)$, where $B$ denotes the unit ball bundle. The family will turn out to be invertible over the spherical bundle $S\left(T^{*} X \times \mathbb{R}\right)$. Therefore, its analytic index is in the following relative group

$$
K\left(B\left(T^{*} X \times \mathbb{R}\right), S\left(T^{*} X \times \mathbb{R}\right)\right) \simeq K\left(T^{*} X \times \mathbb{R}\right)
$$


The desired family of elliptic operators denoted by $D(x, \xi, \tau)$ for the parameters $(x, \xi, \tau) \in B\left(T^{*} X \times \mathbb{R}\right)$ corresponds to a family of symbols

$$
\left[2 \sigma(Q)\left(x, y, \xi, \frac{\eta}{|\eta|}\left(1-\xi^{2}-\tau^{2}\right)\right)-1\right]\left(|\xi|+1-\left(\xi^{2}+\tau^{2}\right)\right)+i \tau .
$$

The invertibility of this symbol for $\xi^{2}+\tau^{2} \leq 1$ can be verified by an explicit computation. It is clear that on the unit spheres for $\xi^{2}+\tau^{2}=1$ this symbol is a vector bundle isomorphism independent of $\eta$ :

$$
(2 \sigma(Q)(x, y, \xi, 0)-1)|\xi|+i \tau \text {. }
$$

Hence, the difference construction for the family of elliptic symbols (27) is an element of the group $K\left(T^{*} \partial M \times \mathbb{R}\right)$.

One can show that this difference construction for the family $D(x, \xi, \tau)$ coincides with element (26). Hence, by the Atiyah-Singer formula for families we obtain the desired expression in analytic terms:

$$
\pi_{!}\left[\left.\sigma(D)\right|_{\partial M}\right]=\text { ind }[D(\cdot, \cdot, \cdot)] \in K^{1}\left(T^{*} X\right)
$$

where

$$
D(x, \xi, \tau)=\left[2 \sigma(Q)\left(x, y, \xi, \frac{\widehat{\eta}}{|\eta|}\left(1-\xi^{2}-\tau^{2}\right)\right)-1\right]\left(|\xi|+1-\left(\xi^{2}+\tau^{2}\right)\right)+i \tau
$$

and the hat means that we have a family of operators in the fibers.

We will show that this family of elliptic operators is homotopic to an invertible family. Therefore, the index is zero in this case.

Denote by $Q(\xi, \tau)$ the following family of pseudodifferential operators in the fibers

$$
Q(\xi, \tau)=\sigma(Q)\left(x, y, \xi, \frac{\widehat{\eta}}{|\eta|}\left(1-\xi^{2}-\tau^{2}\right)\right) .
$$

On the sphere $\xi^{2}+\tau^{2}=1$ for $\xi \neq 0$ this is a family of projections, while for other values of the parameters $Q(\xi, \tau)$ is only an almost-projection. One can verify that this property implies that (29) is elliptic on $B\left(T^{*} X \times \mathbb{R}\right)$ and invertible on $S\left(T^{*} X \times \mathbb{R}\right)$. Let us define the homotopy $D_{\varphi}(x, \xi, \tau)$ by changing this family $Q(\xi, \tau)$ only:

$$
D_{\varphi}(x, \xi, \tau)=\left(2 Q_{\varphi}(\xi, \tau)-1\right)\left(|\xi|+1-\left(\xi^{2}+\tau^{2}\right)\right)+i \tau
$$

such that $Q_{\varphi}(\xi, \tau)$ satisfies the above mentioned property. The homotopy $Q_{\varphi}(\xi, \tau), \varphi \in$ $[0, \pi / 2]$ is defined on the spheres as

$$
Q_{\varphi}(\xi, \tau)=q_{X, \varphi}\left(\frac{\xi}{|\xi|}\right), \quad \text { for } \quad \xi^{2}+\tau^{2}=1
$$


(the family $q_{X, \varphi}$ was defined in (25)), while inside the balls for $\xi^{2}+\tau^{2}<1$ it suffices to define a homotopy of the corresponding symbols:

$$
\sigma\left(Q_{\varphi}\right)(\xi, \tau)=q_{\partial M, \varphi}\left(\xi, \eta\left(1-\xi^{2}-\tau^{2}\right)\right)
$$

(the principal symbols $q_{\partial M, \varphi}$ were defined in (24)). For brevity we omit the geometric variables $x, y$ in the formulas.

One can verify that for this choice of $Q_{\varphi}$ the operator in (30) is elliptic for $\xi^{2}+\tau^{2} \leq 1$ and is an isomorphism for $\xi^{2}+\tau^{2}=1$.

At the end of the homotopy for $\varphi=\pi / 2$ the operator $Q_{\varphi}(\xi, \tau)$ is a pseudodifferential projection that does not depend on $\xi$ and $\tau$. Hence, for $\varphi=\pi / 2$ the family $D_{\varphi}(x, \xi, \tau)$ is invertible on the entire space $B\left(T^{*} X \times \mathbb{R}\right)$. Together with $(\sqrt{28})$ this yields the desired:

$$
\pi_{!}\left[\left.\sigma(D)\right|_{\partial M}\right]=\left[\text { ind } D_{\frac{\pi}{2}}(\cdot, \cdot, \cdot)\right]=0 \text {. }
$$

Example 4. Similar to Example 3, suppose that $\partial M^{4 k}=X^{\text {odd }} \times Y^{e v}$ and consider the projection

$$
X^{\text {odd }} \times Y^{e v} \stackrel{\pi}{\longrightarrow} X^{\text {odd }}
$$

with an even-dimensional fiber $Y^{e v}$.

Now the Hirzebruch operator $D_{M}$ acquires the form

$$
\frac{\partial}{\partial t}+\left(\begin{array}{cc}
D_{X} & D_{Y}^{*} \\
D_{Y} & -D_{X}
\end{array}\right)
$$

In contrast with the previous Example 3, the obstruction does not vanish in this case.

\section{Lemma 1.}

$$
\pi_{!}\left[\left.\sigma(D)\right|_{\partial M}\right]=\text { ind } D_{Y}\left[\sigma\left(D_{X}\right)\right] \in K^{1}\left(T^{*} X\right) .
$$

Proof. This follows from the index formula for families.

According to this Lemma, the Hirzebruch operator has no Fredholm boundary value problems if ind $D_{Y} \neq 0$. When ind $D_{Y}=0$, consider a boundary value problem

$$
\left\{\begin{array}{l}
{\left[\frac{\partial}{\partial t}+\left(\begin{array}{cc}
D_{X} & D_{Y}^{*} \\
D_{Y} & -D_{X}
\end{array}\right)\right]\left(\begin{array}{l}
u \\
v
\end{array}\right)=\left(\begin{array}{l}
f_{1} \\
f_{2}
\end{array}\right),} \\
\left.u\right|_{\partial M}+\left.D_{Y}^{*}\left(\triangle_{Y}+1\right)^{-1} v\right|_{\partial M}=g .
\end{array}\right.
$$

Proposition 3. Boundary value problem (31) has the Fredholm property.

Proof. The check of the ellipticity of the boundary condition is similar to the one in Example 3 and is left to the reader. 


\section{Appendix}

In this appendix we prove two important technical results. First, we establish the boundedness in the Sobolev spaces for a class of operators with operator-valued symbols. Second, we prove the composition formula from Section 3.

Theorem 7. Suppose that an operator-valued symbol $\widehat{p}(x, \xi) \in \Psi^{d}(Y)$ of order $d$ is defined for $x \in \mathbb{R}^{n}$, vanishes outside a compact set and satisfies the estimates

$$
\left\|\left(1+\Delta_{X}\right)^{N}\left(\Delta_{Y}+\xi^{2}\right)^{(s-d) / 2} \widehat{p}(x, \xi)\left(\Delta_{Y}+\xi^{2}\right)^{-s / 2}\right\|_{L^{2}(Y) \rightarrow L^{2}(Y)} \leq C_{p}
$$

uniformly in $x$ and $\xi$ for some $N>(n+|s-d|) / 2$. Then the operator

$$
P: C^{\infty}\left(\mathbb{R}^{n} \times Y\right) \rightarrow C^{\infty}\left(\mathbb{R}^{n} \times Y\right)
$$

with operator-valued symbol $\hat{p}$ extends to an operator of order $d$ in the Sobolev spaces and an estimate of its norm is valid

$$
\|P\|_{H^{s}\left(\mathbb{R}^{n} \times Y\right) \rightarrow H^{s-d}\left(\mathbb{R}^{n} \times Y\right)} \leq C_{p} \cdot C(s, d),
$$

where the constant $C(s, d)$ does not depend on the operator.

Proof. Let us estimate the norm $\|P u\|_{H^{s-d}\left(\mathbb{R}^{n} \times Y\right)}$. In terms of the Fourier transform in $x$ this norm can be represented as

$$
\|P u\|_{H^{s-d}\left(\mathbb{R}^{n} \times Y\right)}^{2}=\left\|\left(\Delta_{Y}+\zeta^{2}\right)^{(s-d) / 2} \widehat{P u}(\zeta)\right\|_{L^{2}\left(\mathbb{R}^{n}, L^{2}(Y)\right)}^{2} .
$$

Since

$$
P u=\int e^{i x \xi} \widehat{p}(x, \xi) \widehat{u}(\xi) d \xi
$$

we obtain that

$$
\widehat{P u}(\zeta)=\int \widetilde{\widehat{p}}(\zeta-\xi, \xi) \widehat{u}(\xi) d \xi
$$

where $\widetilde{p}$ denotes the Fourier transform in $x$ of the operator symbol. Using this expression, we obtain

$$
\|P u\|_{H^{s-d}\left(\mathbb{R}^{n} \times Y\right)}^{2}=\left\|\int\left(\Delta_{Y}+\zeta^{2}\right)^{(s-d) / 2} \widetilde{\widehat{p}}(\zeta-\xi, \xi) \widehat{u}(\xi) d \xi\right\|_{L^{2}\left(\mathbb{R}^{n}, L^{2}(Y)\right)}^{2} .
$$

This shows that $\|P u\|_{H^{s-d}\left(\mathbb{R}^{n} \times Y\right)}^{2}$ is equal to

$$
\left\|\int\left(\Delta_{Y}+\zeta^{2}\right)^{(s-d) / 2} \widetilde{p}(\zeta-\xi, \xi)\left(\Delta_{Y}+\xi^{2}\right)^{-s / 2}\left(\Delta_{Y}+\xi^{2}\right)^{s / 2} \widehat{u}(\xi) d \xi\right\|_{L^{2}\left(\mathbb{R}^{n}, L^{2}(Y)\right)}^{2} .
$$


Let us now estimate the norm of the product in this formula. Consider first the term

$$
A(\xi, \zeta)=\left\|\left(\frac{\Delta_{Y}+\zeta^{2}}{\Delta_{Y}+\xi^{2}}\right)^{(s-d) / 2}\left(\Delta_{Y}+\xi^{2}\right)^{(s-d) / 2} \widetilde{p}(\zeta-\xi, \xi)\left(\Delta_{Y}+\xi^{2}\right)^{-s / 2}\right\|_{L^{2}(Y) \rightarrow L^{2}(Y)} .
$$

The estimate (32) of the theorem implies that

$$
A(\xi, \zeta) \leq \text { Const }\left\|\left(\frac{\Delta_{Y}+\zeta^{2}}{\Delta_{Y}+\xi^{2}}\right)^{(s-d) / 2}\right\|_{L^{2}(Y) \rightarrow L^{2}(Y)} \cdot C_{p} \frac{1}{\left(1+|\zeta-\xi|^{2}\right)^{N}} .
$$

On the other hand, the first term can be estimated by a Peetre type inequality

$$
\left\|\left(\frac{\Delta_{Y}+\zeta^{2}}{\Delta_{Y}+\xi^{2}}\right)^{(s-d) / 2}\right\|_{L^{2}(Y) \rightarrow L^{2}(Y)} \leq \operatorname{Const}\left(1+|\zeta-\xi|^{2}\right)^{|s-d| / 2} .
$$

Thus, the term $A(\xi, \zeta)$ is estimated as

$$
A(\xi, \zeta) \leq \text { Const } \cdot C_{p} \frac{1}{\left(1+|\zeta-\xi|^{2}\right)^{N-|s-d| / 2}} .
$$

We are now in a position to estimate (33)

$$
\|P u\|_{H^{s-d}\left(\mathbb{R}^{n} \times Y\right)}^{2} \leq\left\|\int A(\xi, \zeta)\right\|\left(\Delta_{Y}+\xi^{2}\right)^{s / 2} \widehat{u}(\xi)\left\|_{L^{2}(Y)} d \xi\right\|_{L^{2}\left(\mathbb{R}^{n}\right)}^{2} .
$$

This gives

$$
\|P u\|_{H^{s-d}\left(\mathbb{R}^{n} \times Y\right)}^{2} \leq \text { Const } \cdot C_{p}\|u\|_{H^{s}\left(\mathbb{R}^{n} \times Y\right)}^{2} \cdot \int \frac{d \zeta}{\left(1+|\zeta|^{2}\right)^{N-|s-d| / 2}}
$$

where, for $N>(n+|s-d|) / 2$ the last integral converges. This proves the boundedness of $P$ and the corresponding norm estimate.

Proof of the composition formula. The rest of the appendix contains the proof of the composition formula

$$
\widehat{\sigma}_{1} \widehat{\sigma}_{2} \equiv \widehat{\sigma_{1} \sigma_{2}}
$$

for two symbols $\sigma_{1}, \sigma_{2} \in \Sigma(M, \pi)$. The comparison is valid modulo a compact operator in the scale of Sobolev spaces. 
Lemma 2. If the composition formula is valid for all $\sigma_{1} \in \Sigma_{0}, \sigma_{2} \in \Sigma(M, \pi)$. Then it is true in general.

Proof. Consider a pair of symbols $\sigma_{1}, \sigma_{2} \in \Sigma(M, \pi)$. Choose an approximation of $\sigma_{1}$ by an element $\sigma_{\varepsilon}$ of the dense subalgebra $\Sigma_{0} \subset \Sigma(M, \pi)$ (see Proposition 1). Then the difference of the corresponding operators is denoted by

$$
A_{\varepsilon}=\widehat{\sigma}_{1}-\widehat{\sigma}_{\varepsilon}
$$

and has norm less than $\varepsilon$ in any given Sobolev space, provided the approximation is chosen appropriately. Thus, we obtain

$$
\widehat{\sigma}_{1} \widehat{\sigma}_{2}=\left(\widehat{\sigma}_{\varepsilon}+A_{\varepsilon}\right) \widehat{\sigma}_{2}
$$

By the assumption of the Lemma we have

$$
\left(\widehat{\sigma}_{\varepsilon}+A_{\varepsilon}\right) \widehat{\sigma}_{2}=\widehat{\sigma_{\varepsilon} \sigma_{2}}+A_{\varepsilon} \widehat{\sigma}_{2}+K_{\varepsilon}
$$

(here $K_{\varepsilon}$ is a compact operator). Hence,

$$
\widehat{\sigma}_{1} \widehat{\sigma}_{2}=\widehat{\sigma_{1} \sigma_{2}}+A_{\varepsilon} \widehat{\sigma}_{2}+\widehat{\sigma}^{\prime}+K_{\varepsilon}
$$

where $\sigma^{\prime}$ denotes the symbol $\left(\sigma_{\varepsilon}-\sigma_{1}\right) \sigma_{2}$ with a small norm. As we let $\varepsilon \rightarrow 0$, this equality shows that the difference

$$
\widehat{\sigma_{1} \sigma_{2}}-\widehat{\sigma}_{1} \widehat{\sigma}_{2}
$$

is a norm limit of a family of compact operators $K_{\varepsilon}$. Therefore, the difference is compact as well.

Lemma 3. The composition formula is valid for all symbols $\sigma_{1} \in \Sigma_{0}, \sigma_{2} \in \Sigma(M, \pi)$. Moreover, the error term has order -1 in the Sobolev spaces.

Proof. Obviously, it is sufficient to prove the formula when $\sigma_{1}$ either corresponds to an operator with a smooth symbol or a family of pseudodifferential operators in the fibers. We consider the two possibilities separately.

1) Let

$$
\sigma_{1}=(a(x, y, \xi, \eta), a(x, y, \xi, 0))
$$

correspond to a smooth symbol $a(x, y, \xi, \eta)$ and $\sigma_{2}=\left(p_{M}, p_{X}\right)$ be a general symbol. The composition of the corresponding operators has the form

$$
\left.\widehat{a}\left(\widehat{p}+\widehat{p_{X}-\widehat{\widetilde{p}}_{M}}\right)\right) .
$$


The desired composition formula will be proved if we show that

$$
\widehat{a} \widehat{p}_{M} \equiv \widehat{a p}_{M}
$$

and for a compact operator-valued symbol $q_{X}=p_{X}-\widehat{\widetilde{p}}_{M}$ a similar comparison is valid

$$
\widehat{a} \widehat{q}_{X} \equiv \widehat{\widetilde{a} q_{X}}
$$

a) To prove (34) we have to show that the usual composition formula for $\psi D O$ 's on $M$ remains valid in this case (since the expressions for the operators in (34) do not contain additional operator-valued components).

The usual proof of the composition formula (e.g., see [14], p. 32) can be repeated verbatim to establish (34). Moreover, the composition formula is valid modulo an operator of order -1 in the scale of Sobolev spaces.

b) To prove (35) let us represent operator $\widehat{a}$ as an operator on $X$ with an operatorvalued symbol (see [7]):

$$
a^{\prime}(x, \xi)=a\left(x, y, \xi,-i \frac{\partial}{\partial y}\right): L^{2}\left(Y_{x}\right) \longrightarrow L^{2}\left(Y_{x}\right)
$$

Let us first prove that the composition formula is satisfied modulo a compact operator in $L^{2}(M)$. From the composition formula for operators with operator-valued symbols (see [7]) we obtain

$$
\widehat{a} \widehat{q}_{X}-\widehat{a^{\prime} q_{X}} \equiv 0 .
$$

On the other hand, the right-hand side of the desired formula (35) in this case is equal to $\widehat{\widetilde{a} q_{X}}$.

To prove that the two expressions $\widehat{a^{\prime} q_{X}}$ and $\widehat{\widetilde{a} q_{X}}$ differ by a compact operator, it suffices to show (see [7]) that the $L^{2}$-norm of the compact operator-valued symbol

$$
\left[a\left(x, y, \xi,-i \frac{\partial}{\partial y}\right)-a(x, y, \xi, 0)\right] q_{X}(x, \xi)
$$

tends to zero as $|\xi| \rightarrow \infty$. Indeed, the symbol of the operator in the square brackets is equal to $a(x, y, \xi, \eta)-a(x, y, \xi, 0)$ and can be estimated as:

$$
|a(x, y, \xi, \eta)-a(x, y, \xi, 0)| \leq C \frac{|\eta|}{|\xi|+|\eta|+1} .
$$

A similar estimate is valid for the derivatives in $y$ (with a possibly different constant $C$ ).

From this estimate it follows that the norm of the corresponding operator is estimated as follows

$$
\left\|a\left(x, y, \xi,-i \frac{\partial}{\partial y}\right)-a(x, y, \xi, 0)\right\|_{H^{1}\left(Y_{x}\right) \rightarrow H^{0}\left(Y_{x}\right)} \leq \frac{C^{\prime}}{1+|\xi|}
$$


Hence, the norm of the symbol (37) in $L^{2}$ is bounded by:

$$
\begin{aligned}
& \left\|\left[a\left(x, y, \xi,-i \frac{\partial}{\partial y}\right)-a(x, y, \xi, 0)\right] \cdot q_{X}(\xi)\right\|_{L^{2}\left(Y_{x}\right) \rightarrow L^{2}\left(Y_{x}\right)} \leq \\
& \left\|q_{X}\right\|_{L^{2}\left(Y_{x}\right) \rightarrow H^{1}\left(Y_{x}\right)} \times\left\|a\left(x, y, \xi,-i \frac{\partial}{\partial y}\right)-a(x, y, \xi, 0)\right\|_{H^{1}\left(Y_{x}\right) \rightarrow L^{2}\left(Y_{x}\right)} \leq \frac{C^{\prime \prime}}{1+|\xi|}
\end{aligned}
$$

Hence, it tends to zero as desired. This proves that the composition formula is valid in this case modulo a compact operator in $L^{2}(M)$. However, we need to prove a stronger statement that the error term has order -1 in the Sobolev scale. To prove this, it suffices to show that the difference in (36) and the operator with symbol (37) have order -1 .

First, similar to the proof of Theorem 1, one can obtain for the symbol in (37) the estimate of the form

$$
\left\|\left(\sqrt{\Delta_{Y}}+|\xi|\right)^{s+1}\left(a^{\prime}-\widetilde{a}\right) q_{X}\left(\sqrt{\Delta_{Y}}+|\xi|\right)^{-s}\right\|_{L^{2}\left(Y_{x}\right) \rightarrow L^{2}\left(Y_{x}\right)} \leq C
$$

and a similar estimate for its derivatives in $x$. Thus, similar to the Theorem 0 , this proves that the symbol $\left(a^{\prime}-\widetilde{a}\right) q_{x}$ gives an operator of order -1 in the Sobolev scale.

Second, to estimate the difference (36) one should estimate the corresponding error term in the composition formula (e.g., see [14], p. 32). This can also be done. The details are left to the reader.

2) Let us now verify the composition formula for a symbol

$$
\sigma_{1}=(b(x, y, \eta), B(x))
$$

of a family of operators $B(X)$ in the fibers and $\sigma_{2}=\left(p_{M}, p_{X}\right)$ as before. In this case the proof of the composition formula amounts to verifying the two comparisons (here $q_{X}=p_{X}-\widehat{\widetilde{p}}_{M}$ as before)

$$
\begin{gathered}
B \widehat{q_{X}} \equiv \widehat{B q_{X}}, \\
B \widehat{p_{M}} \equiv \widehat{b p_{M}}+\widehat{B \widehat{\widetilde{p}}_{M}-\widehat{b \tilde{p}_{M}}} .
\end{gathered}
$$

Concerning the first composition, it is easy to see that it is satisfied exactly.

Let us establish comparison (39). To this end, we rewrite the terms $B \widehat{p}_{M}$ and $\widehat{b p}_{M}$ as operators on the base $X$ with operator-valued symbols. We introduce the following notation. For a symbol $\sigma(x, y, \xi, \eta)$ denote the operator-valued symbol $\sigma(x, y, \xi,-i \partial / \partial y)$ by $\sigma^{\prime}$. Then (39) can be rewritten as

$$
\widehat{B p_{M}^{\prime}} \equiv \widehat{\left(b p_{M}\right)^{\prime}}+\widehat{q}_{X}
$$


where the operator symbol $q_{X}$ is equal to

$$
q_{X}=B \widehat{\widetilde{p}}_{M}-\widehat{\widetilde{p}}_{M}
$$

Thus, to prove that the comparison (39) is valid modulo a compact operator, it suffices to show that the operator-valued symbol

$$
B p_{M}^{\prime}-\left(b p_{M}\right)^{\prime}-B \widehat{\widetilde{p}}_{M}+\widehat{\widetilde{p}}_{M}
$$

is a compact operator for all $(x, \xi) \in T^{*} X$ and its norm tends to zero as $|\xi| \rightarrow \infty$.

First of all, the compactness of (40) is easy to obtain taking the symbols of the operators involved:

$$
\sigma\left(B p_{M}^{\prime}-\left(b p_{M}\right)^{\prime}-B \widehat{\widetilde{p}}_{M}+\widehat{b \widetilde{p}_{M}}\right)=0
$$

Here we omitted for brevity the variables $x, y$ in the symbols.

Let us now show that the norm of the symbol (40) tends to zero as $|\xi| \rightarrow \infty$. To this end, we rewrite this symbol in the form

$$
B\left(p_{M}-\widetilde{p}_{M}\right)^{\prime}-\left(b\left(p_{M}-\widetilde{p}_{M}\right)\right)^{\prime}=\left(B-b^{\prime}\right)\left(p_{M}-\widetilde{p}_{M}\right)^{\prime}+b^{\prime}\left(p_{M}-\widetilde{p}_{M}\right)^{\prime}-\left(b\left(p_{M}-\widetilde{p}_{M}\right)\right)^{\prime} .
$$

For the difference $p_{M}-\widetilde{p}_{M}$ the following estimate can be obtained

$$
\left|p_{M}(\xi, \eta)-\widetilde{p}_{M}(\xi, \eta)\right| \leq C \frac{|\eta|}{|\xi|+|\eta|+1} .
$$

Similar to the previous part of the proof this shows that the operator-valued symbol $\left(B-b^{\prime}\right)\left(p_{M}-\widetilde{p}_{M}\right)^{\prime}$ in (42) tends to zero for $\xi \rightarrow \infty$. Let us estimate the remaining term

$$
b^{\prime}\left(p_{M}-\widetilde{p}_{M}\right)^{\prime}-\left(b\left(p_{M}-\widetilde{p}_{M}\right)\right)^{\prime}
$$

by means of the usual composition formula on the fiber. It is a pseudodifferential operator of order -1 with a symbol $C(\xi, \eta)$ estimated as

$$
|C(\xi, \eta)| \leq \frac{C_{1}}{|\xi|+|\eta|+1}
$$

Thus, the norm of the corresponding operator-valued symbol tends to zero as $|\xi| \rightarrow \infty$. The proof that the composition formula is valid modulo an operator of order -1 can be obtained similar to the previous part of the proof.

This completes the proof of the composition formula. 


\section{References}

[1] M.F. Atiyah and R. Bott. A Lefschetz fixed point formula for elliptic complexes. I. Ann. of Math., 86, 1967, 374-407.

[2] A. Savin and B. Sternin. Index defects in the theory of nonlocal boundary value problems and the $\eta$-invariant. Univ. Potsdam, Institut für Mathematik, Potsdam, 2001. Preprint N 01/31.

[3] M.F. Atiyah and I.M. Singer. The index of elliptic operators IV. Ann. Math., 93, 1971, 119-138.

[4] B.-W. Schulze. Pseudo-Differential Boundary Value Problems, Conical Singularities, and Asymptotics, volume 4 of Mathematics Topics. Akademie Verlag, Berlin, 1994.

[5] B.A. Plamenevskij and G.V. Rozenblyum. Pseudodifferential operators with discontinuous symbols: $K$-theory and the index formula. Funct. Anal. Appl., 26, No. 4, 1992, 266-275.

[6] J. Kohn and L. Nirenberg. An algebra of pseudo-differential operators. Comm. Pure Appl. Math., 18, 1965, 269-305.

[7] G. Luke. Pseudodifferential operators on hilbert bundles. J. Diff. Equation, 12, 1972, $566-589$.

[8] S. Rempel and B.-W. Schulze. Index Theory of Elliptic Boundary Problems. Akademie-Verlag, Berlin, 1982.

[9] A.Yu. Savin and B.Yu. Sternin. Elliptic operators in even subspaces. Matem. sbornik, 190, No. 8, 1999, 125-160. English transl.: Sbornik: Mathematics 190, N 8 (1999), p. 1195-1228; http://xxx.lanl.gov/abs/math/9907027.

[10] B.-W. Schulze, B. Sternin, and V. Shatalov. On general boundary value problems for elliptic equations. Math. Sb., 189, No. 10, 1998, 145-160. English transl.: Sbornik: Mathematics 189, N 10 (1998), p. 1573-1586.

[11] L. Hörmander. The Analysis of Linear Partial Differential Operators. III. SpringerVerlag, Berlin Heidelberg New York Tokyo, 1985.

[12] R.S. Palais. Seminar on the Atiyah-Singer index theorem. Princeton Univ. Press, Princeton, NJ, 1965.

[13] M. Atiyah, V. Patodi, and I. Singer. Spectral asymmetry and Riemannian geometry I. Math. Proc. Cambridge Philos. Soc., 77, 1975, 43-69. 
[14] Yu. Egorov and B.-W. Schulze. Pseudo-Differential Operators, Singularities, Applications. Birkhäuser, Boston, Basel, Berlin, 1997. 